\title{
Kinetics Of The Enzymatic Transesterification Of Tuna Oil Catalyzed By Immobilized Candida Rugose Lipase To Produce Structured Lipid High In Omega-3 Fatty Acids
}

\author{
Rizka Amalia ${ }^{1, *}$, Wahyuningsih Wahyuningsih ${ }^{1}$, RTD Wisnu Broto $^{1}$, Mohamad Endy Yulianto ${ }^{1}$, Hafizh Rama \\ Devara $^{2}$, and Dwi Yunita Indah Sari ${ }^{2}$ \\ ${ }^{1}$ Diploma Program of Chemical Engineering, Vocational School, Diponegoro University, Semarang - Indonesia \\ ${ }^{2}$ Student of Diploma Program of Chemical Engineering, Vocational School, Diponegoro University, Semarang - Indonesia
}

\begin{abstract}
Structured lipid containing Medium Chain of Fatty Acid (MCFA) at outer position and Poly-Unsaturated Fatty Acids (PUFA) at sn-2 position has nutritional value and excellent absorption. In this research, structured lipids was synthesized directly through enzymatic acidolysis between fish oil and lauric acid and catalyzed by specific lipase from immobilized 1.3 Candida rugose. The kinetics of enzymatic transesterification reactions catalyzed by immobilized Candida rugose was studied. To obtain the optimal condition, the factor substrate ratio of fish oil : lauric acid and reaction time were investigated. Simple mathematical model for DAG synthesis through transesterification mechanisms have been developed. The results showed that the parameters obtained had a good sensitivity. It was found that the kinetic model well describes the behavior of the reaction as the influence of the initial ratio of reactants.
\end{abstract}

Keywords: kinetics; MCFA; catalyst; Candida rugose; tuna oil.

\section{Introduction}

Synthesis of structured lipids have been developed rapidly over the past decade by modifying lipids, especially for improving the functional and nutritional properties of a fat or oil. Structured lipids containing Medium Chain Fatty Acid $\left(\mathrm{C}_{6}-\mathrm{C}_{12}\right)$ at outer position and Poly-Unsaturated Fatty Acids (PUFA) at sn-2 position has nutritional value and excellent absorption [1]. Residues of medium chain are more rapidly hydrolyzed in the digestive tract, produces fatty acids which is quickly absorbed and has a high source of energy for human body. PUFA was absorbed as 2-Monoglycerides which is most rapidly absorbed among the others PUFA's derivative compounds. Structured lipids with saturated residue at outer position and PUFA at sn-2 position were also more resistant to oxidation [2]. In this research the production of structured lipids rich in omega-3 efficiently will be conducted.

The behavior of the enzymatic transesterification reaction was studied by determine the simple mathematical model of transesterification The model proposed was expected to be used for the study of the reaction's behavior at different operating conditions. Previous studies of the the kinetics of the esterification reaction were used as a reference of this research : Rosu [3] conducted a study of Synthetic DAG Esterification with biocatalyst Lipoze RM IM, and proposed a kinetic model as shown in Figure 1. This model was developed based on the esterification of free fatty acids and glycerol in the solvent-free systems which followed by simultaneous removal of water. Furthermore Watanabe [4] conducted an experiments with a similar system reactions on different reactors, ie Continuous Packed Bed Reactor using a Vacuum Pump. The process developed by Watanabe has been used to produce DAG oil on an industry in Japan.

Simulation which can explain the behavior of substrate is required to understand the esterification reaction of free fatty acids and glycerol to produce DAG oil. In this study, a simulation performed by using a kinetic model based on the proposed mechanism by Hermansyah [5].

\section{Methodology}

Transesterification reaction following the method of Watanabe [4]. Oleic acid as the source of fatty acids; the initial concentration of tuna oil : $1,29 \mathrm{M}$; the initial concentration of oleic acid : $2,59 \mathrm{M}$; reaction temperature at $50^{\circ} \mathrm{C}$, the enzyme used is immobilized Candida rugose with $5 \%$ concentration (dry base), the removal of water carried out under vacuum of $3 \mathrm{mmHg}$.

Validation was done by fit the experimental data of concentration to a proposed model [6]. The data used are the concentration of FA, 1-MAG, 2-MAG, 1,2DAG, 1,3-DAG and TAG. The solution of the differential equation was calculate numerically using the $4^{\text {th }}$ order Runge-Kutta method with time increment at 0.1 hours. Figure 2 shows the validation path to determine the estimated value of the kinetic constants.

* Corresponding author: riz.chemicalia@gmail.com 


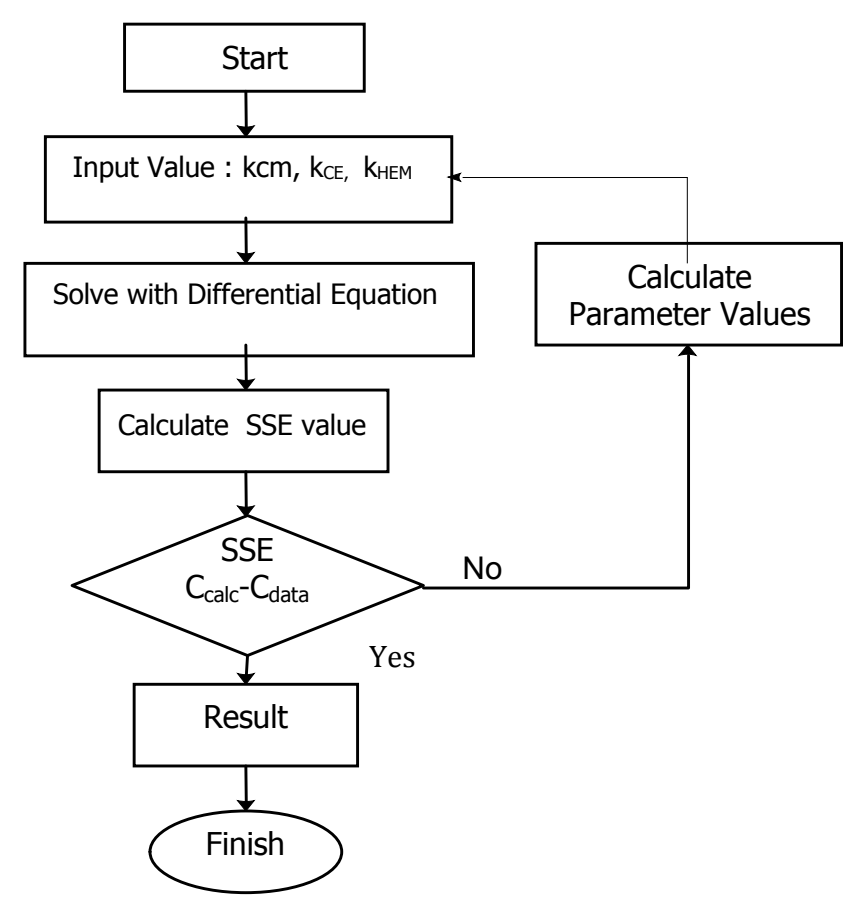

Fig 1 Flowchart of model validation

\section{Results and Discussion}

The difference between the proposed model and the literature model is seen in the mechanism of the reaction. Reaction mechanism from the literature was a reversible [6]. This is based on different assumptions about the absence of equilibrium condition in the system. The literature model assumes that the equilibrium reaction was not occurred as a result of simultaneous water discharge of the system, so that the model model can calculate the rate of water removal [7].

While on the proposed model, the rate of water removal was ignored. It was considerate that the water removed from the system continuously causes no reverse reaction which leads to the occurrence of oil hydrolysis, or in other words the reaction was irreversible [2].

From the data obtained, the boundary condition and the value of $\mathrm{K}$ at various temperature can be determined.

$K=\frac{C_{T Q^{x_{E}}}\left(3 C_{T O^{x_{\theta}}}\right)^{3}}{C_{T Q}\left(1-x_{\theta}\right)\left(R_{M T}-3 x_{\theta}\right)^{3}}$

Data from the study are as follows:

At $\mathrm{t}=0$ :

$\mathrm{C}_{\mathrm{T}}=$ the initial concentration of tuna fish oil $=2.59$ $\mathrm{M}$;

$\mathrm{R}_{\mathrm{MT}}=$ concentration ratio of the Monoasyl Glycerol :

Triacyl Glycerol;

$\chi_{\mathrm{e}} \quad=$ mole fraction of Triasil Glycerol at equilibrium.

The calculated value of $\mathrm{K}$ from equation (1) at various temperature are as follows :
Table 1. The value of $\mathrm{R}_{\mathrm{MT}}$ and $\mathrm{K}$ at various temperature

\begin{tabular}{|c|c|c|c|c|c|}
\hline $\mathrm{T}\left({ }^{\circ} \mathrm{C}\right)$ & $\%_{\mathrm{TG}}$ & $\chi_{\mathrm{e}} \mathrm{TG}$ & $\chi_{\mathrm{e}} \mathrm{MG}$ & $\mathrm{R}_{\mathrm{MT}}$ & $\mathrm{K}$ \\
\hline 30 & 0.6 & 0.006 & 0.0052 & 0.8667 & $1 \times 10^{-6}$ \\
\hline 40 & 0.99 & 0.0099 & 0.0097 & 0.9798 & $5.3 \times 10^{-6}$ \\
\hline 50 & 1.78 & 0.0178 & 0.0165 & 0.927 & $7.19 \times 10^{-5}$ \\
\hline 60 & 2.62 & 0.0262 & 0.0203 & 0.7748 & $6.72 \times 10^{-4}$ \\
\hline 70 & 3.08 & 0.0308 & 0.0265 & 0.86 & $9.61 \times 10^{-4}$ \\
\hline
\end{tabular}

The results from Table 1 were then applied to the equation (2)

$$
\ln \frac{K}{K_{298}}=\frac{-\Delta H^{\circ}}{R}\left(\frac{I}{T}-\frac{I}{298}\right)
$$

To facilitate the determination of the approach method to define the equilibrium equation, the variable $\mathrm{T}$ will be taken in the form $1 / \mathrm{T}$, while the variable $\mathrm{K}$ is brought into the form $\ln \mathrm{K}$. The relationship between the two variables can be seen:

Table 2 The Value of $\ln \mathrm{K}$ at various Temperature

\begin{tabular}{|l|l|l|l|}
\hline $\boldsymbol{T}\left({ }^{\circ} \boldsymbol{C}\right)$ & $\mathbf{1} / \mathbf{T}\left({ }^{\circ} \mathbf{C}^{-\mathbf{1}}\right)$ & $\mathbf{K}$ & $\mathbf{l n} \mathbf{K}$ \\
\hline 30 & 0.033 & $1 \times 10^{-6}$ & -13.815 \\
\hline 40 & 0.025 & $5.3 \times 10^{-6}$ & -12.147 \\
\hline 50 & 0.02 & $7.19 \times 10^{-5}$ & -9.539 \\
\hline 60 & 0.0167 & $6.72 \times 10^{-4}$ & -7.304 \\
\hline 70 & 0.0143 & $9.61 \times 10^{-4}$ & -6.947 \\
\hline
\end{tabular}

By plotting the value of $1 / \mathrm{T}$ vs $\ln \mathrm{K}$, showing a linear relation. Therefore, the equation (14) can be brought into a linear form

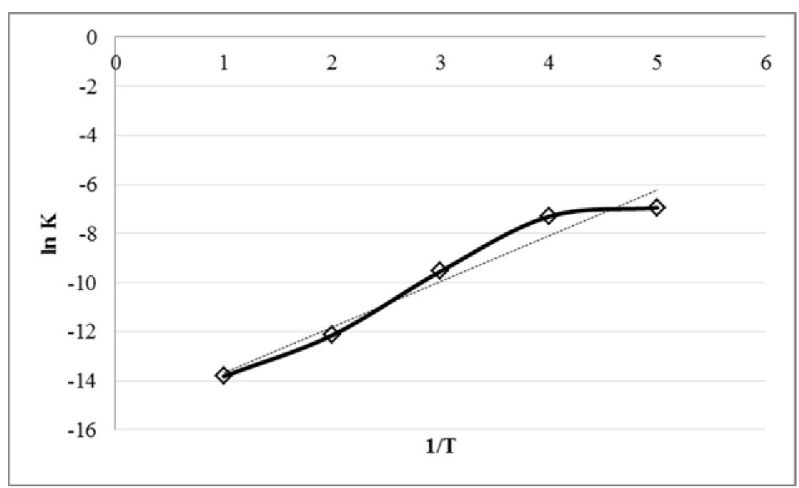

Fig 2 Plot of $\ln \mathrm{K}$ vs $1 / \mathrm{T}$

Referring to the value of kinetic constant in Table 1 , the rate of 1,3 DAG formation was much greater than the rate of $\mathrm{TAG}$.

The sensitivity of kinetic parameters obtained from the model can be found by changing one of the kinetic value become half or twice from the initial value without changing the other constant. Thus, the deviation was observed to determine whether the parameter have a good sensitivity if the changing in value results in a significant error. Table 2 shows the results of the kinetic sensitivity analysis for the proposed model [1].

A simulation was conducted to determine the effect of reaction conditions towards the concentration of DAG. In this study, the variations only can be done on the initial concentration ratio of lauric acid and tuna fish oil [8]. 
At the beginning of the reaction, 1.3-DAG is rapidly formed due to the more produced of 1-MAG from the reaction of lauric acid with tuna oil. The fast reaction characterized by the increasing of DAG concentration, due to the high activity of enzyme. Stationary conditions may occur as one of the reactants has been reacted completely. Although basically the esterification reaction is a reversible reaction, but assuming the reaction was irreversible can be accepted, it because the system of water removal was formed simultaneously, so the equilibrium was not achieved and the reverse reaction does not occured. Therefore, it can be concluded that stastioner condition occurs due to the one or both reactants were consumed completely.

At the ratio of 2: 1 of fatty acid : glycerol, the stationary conditions does not occured. This may be due to the initial concentration ratio of the reactants appropriate to the stoichiometric ratio between the two reactants for producing DAG ( 2 moles of fatty acid for 1 mole of glycerol). Thus, although at the ratio of fatty acid:glycerol 2:1 does not resulting the highest concentration of DAG, it was the most efficient ratio [9].

\section{Conclusion}

Simple mathematical model for DAG synthesis through transesterification mechanisms have been developed. The results showed that the parameters obtained had a good sensitivity. It was found that the kinetic model well describes the behavior of the reaction as the influence of the initial ratio of reactants.

The basic idea of low carbon society is to cut down all carbon dioxide emissions without endangering any developing needs. Enzymatic transesterification is one of the method for producing biofuels also, which has low emission of carbon. In this way, it will help to achieve a low carbon society.

\section{References}

1. Chiffci, R., K. Furihata, K. Hata, Y. Iwasaki, and T. Yamane. 2009. Two-Step

Enzymatic
Synthesis of Docosahexaenoic Acid-Rich symmetrically Structured TriacilglycerolVia2Monoacylglycerols.Hour. Oil. Chem. Soc. 78 (7): 743-748.

2. Endo, JR, DH Pence, S. Scheinsach, PR D'Amelia, LP Klemann, NH Wilson, and JW Finkey. 2007. Review of Triacilglycerols Digestion, Absorption, and Metabolism With Respect to SalatrimTriacylglycerols. J. Agri. Food. Chem. 42.473-483

3. Rosu, C., H. Austin, L. Porsorske, and J. Gonziez. 2007. Charachteristic of an Immobilized Lipase for the Commercial synthesis of Esther.Hour. Oil. Chem. Soc. 65 (6): 927-935

4. Watanabe, T., Sugiara, M., Sato, M., Yamada, N., And Nakanishi, K., (2004), Diacyl glycerol production in a packed bed bioreactor, Process chemistry 40 , pp 637-643

5. H., Hermansyah, Tania, S., T., Rita, A., and Dawn, A., Simulation esterification reaction of free fatty acids and glycerol to produceoil, diacylglycerol Reactor V0L 13 no 2 , December 2010, p 95-102

6. T. Watanabe, M. Shimizu, M. Sugiura, M. Sato, J. Kohori, N. Yamada, K. Nakanishi, J. Am. Oil Chem. Soc. 80 (2003) 1201.

7. Jeyarani, S., Y. Iwasaki, and CT Hou. 2010. Study of Ethanolysis to 2-MAG by Immobilized Candida Antarctica Lipase and Synthesis of symmetrically StructuredTAG.Hour. Oil. Chem. Soc. 79 (9): 879883

8. Idris, R., K. Furihata, K. Hata, Y. Iwasaki, and T. Yamane.2005.utilization of MediumDependent Reaction Regiospecifity of Candida Antarctica Lipase (Novozyme 435) For The Synthesis of 1,3-Dicapryloyl-2Docosahexaenoyl (Or Eicosapentaenoyl) Glycerol. Hour. Oil. Chem. Soc. 78 (3): 285-289

9. Monisvea A., Y. Shimada, M. Yamamoto, A. Sugihara, T. Nagao, S. Komemushi, and Y. Tominaga. 2009. Enzymatic Synthesis of HighPurity Structured Lipids caprylic acid at a 1.3Positions and Polyunsaturated Fatty Acid at2Position.Hour. Oil. Chem. Soc. 78 (6): 611-616 